\title{
Study of Bioactivities of Holarrhena pubescence Growing in Bangladesh
}

\author{
A K Lutful Kabir ${ }^{1 x}$, Mst. Marium Begum ${ }^{2 x}$ and Tahiya Islam ${ }^{3}$ \\ ${ }^{1}$ Department of Pharmaceutical Technology, Faculty of Pharmacy, University of Dhaka \\ Dhaka-1000, Bangladesh \\ ${ }^{2}$ Department of Pharmacy, East West University, Aftabnagar, Dhaka-1212, Bangladesh \\ ${ }^{3}$ Department of Biomedical Science, Universiti Putra Malaysia, 43400 UPM, Serdang Darul Ehsan \\ Malaysia
}

(Received: 4 December, 2017; Accepted: 28 May, 2018; Published (web): 10 June, 2018)

\begin{abstract}
The stem bark of Holarrhena pubescence was tested for anti-diabetic, analgesic, anti-inflammatory and antidiarrheal activities. After 1, 4 and 12 weeks of treatment protocol in alloxan-induced diabetic rats, it showed very negligible reduction of blood glucose level. In 12 weeks treatment protocol $H$. pubescence demonstrated that, the extract at 300 and $600 \mathrm{mg} / \mathrm{kg}$ bw can reduce blood glucose level by 3 and $4 \mathrm{mmol} / \mathrm{l}$, respectively. Bark extract possessed strong analgesic, anti-diarrheal and mild anti-inflammatory activities. A dose of $600-\mathrm{mg} / \mathrm{kg}$ showed $69.50 \%(\mathrm{p}<0.05)$ of writhing inhibition compared to indomethacin $(73.76 \%)$ for acetic acid-induced analgesic method. In formalin-induced anti-nociception, the extract at 300 and $600 \mathrm{mg} / \mathrm{kg}$ bw showed dose dependent antinociception $(67.42 \%$ and $69.007 \%$, respectively, $\mathrm{p}<0.05)$. The anti-diarrheal study demonstrated significant antidiarrheal potential in a dose dependent manner as demonstrated by reduction of defecation by $64.92 \%$ and $71.43 \%$, for $300-$ and $600-\mathrm{mg} / \mathrm{kg}$ bw, respectively.
\end{abstract}

Key words: Holarrhena pubescence, Anti-diabetic, Analgesic, Anti-inflammatory, Anti-diarrheal.

\section{INTRODUCTION}

Holarrhena pubescence (Buch.-ham.) Wall. (syn- Holarrhena antidysenterica (Roxb. ex Fleming) Wall.) commonly known as kurchi, is a flowering plant of Apocynaceae family of Indian subcontinent and also indigenous to Bangladesh. ${ }^{1}$ Its stem bark has been well reported for antibacterial, antidiarrheal, anti-malarial, muscle relaxant, CNS depressant activities. ${ }^{2-6}$ Seeds of this plant has potentials for free radical scavenging, anti-diabetic, hypoglycemic, antidiuretic, anti-hyperlipi-dimic and in vivo and in vitro anti-urolithic activities. ${ }^{7-12}$ Leaves of $\mathrm{H}$. pubescence are reported for anti-anthelminthic, antiinflammatory, analgesic and antioxidants activities. ${ }^{13-15}$ Seed and bark contain various important alkaloids. For example, pubadysone [11ahydroxy-18,20-oxido-3-oxo-pregna-1,4,17(20)-triene], puboestrene [3-acetoxy-17-oxo-1,3,5 (10)-estratri ene]

Correspondence to: A K Lutful Kabir

Email: lutful@du.ac.bd,

a Both authors contributed equally.

Dhaka Univ. J. Pharm. Sci. 17(1): 131-137, 2018 (June) and pubamide [3,18-dioxo-11a-hydroxycona-1,4diene] have been isolated from the bark of $H$. pubescence. ${ }^{16}$ Bark is enriched with steroidal alkaloids such as conessine, isoconessimine, conessimin, conarrhimin and conimin. These alkaloids could be potential candidates for further development of new drugs against $\mathrm{AD}{ }^{17}$ So we designed our research to find out the ant-diabetic, anti-diarrheal, analgesic and anti-inflammatory activities of stem bark of $\mathrm{H}$. pubescence which are not reported previously. We also used different methods of finding these pharmacological activities.

\section{METHODS}

Chemicals and drugs. Alloxan was purchased from Sigma Chemical Company, USA. Indomethacin and loperamide were procured from Square Pharmaceuticals Ltd. Formalin and acetic acid (Mark - Germany) were used to induce pain. Carrageenan was purchased from Sigma Chemical Co. (USA). All 
other chemicals used in the experiments were generous gifts from Eskeyef Pharmaceutical Company Ltd., Bangladesh. The chemicals were of analytical grade.

Animals. The test rats were collected from the International Centre for Diarrheal Disease Research, Bangladesh (icddr,b). The experiments were performed by using Long-Evans male rats weighing about 200 - 220 gram, aged between 2 -2.5 months. Feeding of animals was done with normal pellet, along with drinking water and they were maintained at natural day and night cycle.

Experimental design. In performing antidiabetic study, after one week of acclimation, the test animals were divided into five major groups. Long Evan male rats (90) were divided into three different time frames to perform 1 week, 4 weeks and 8 weeks treatment protocol. For 1 week protocol, 5 groups had been assigned with total 30 test rats and groups were assigned as normal, control, glibenclamide, and MEHP $300-\mathrm{mg} / \mathrm{kg}$ bw, $600-\mathrm{mg} / \mathrm{kg}$ bw. For 4 weeks and 12 weeks treatment protocol, same grouping had been followed. Besides this, after acclimation for 7 days, Long Evan rats (24) were randomly divided into four groups (normal, standard and two MEHP doses) to analyze acetic acid induced analgesic test. Each group had 6 rats as sample. The same protocol was followed for formalin-induced analgesic, carrageenan induced anti-inflammatory and castor oil induced anti-diarrheal test.

Authentication of plant. The specimen of $H$. pubescence (Buch.-Ham.) Wall. was submitted to the Bangladesh National Herbarium (BNH), Dhaka, Bangladesh where it was given the accession number: DACB-48725.

Preparation of plant extract. After collecting the plant bark, it was cleaned with water and dried at room temperature $\left(24-26^{\circ} \mathrm{C}\right)$ for one week. The completely dried bark was cut into smaller piece and ground to powder, sieved and 400 gram of sieved powder was taken into a conical flask followed by soaking it with $2000 \mathrm{ml}$ of $95 \%$ methanol. After that, the mixture was coarse filtered by a cotton plug followed by Whitman filter paper number 1 . Finally, the filtrate was rotary evaporated using Bibby RE200, Sterilin Ltd., UK at 5-6 rpm and optimum temperature to get a dark brown coloured concentrate that was designated as the crude methanol extract of H. pubescenc.

Phytochemical screening. The methanol extract of bark of $H$. pubescence was qualitatively analysed for identifying the presence of various compounds like tannins, flavonoid, anthraquinone, glycosides terpenoids, alkaloids and phenol.

\section{Assessment of anti-diabetic activity}

Induction of experimental diabetes. The induction of diabetes was performed by allowing single intraperitoneal injection of alloxan monohydrate at a dose of $120 \mathrm{mg} / \mathrm{kg}$ body weight. Alloxan was dissolved in citrate buffer at $\mathrm{pH} 4.5$ and injected immediately within few minutes to avoid degradation. After 72 hours, from the retro-orbital plexus, blood was collected and blood glucose level was determined by using auto analyzer (Microlab 2000) from all surviving rats.

Preparation of test samples. Diabetic rats received treatment with i.p injection of glibenclamide $(1.2 \mathrm{mg} / 70 \mathrm{~kg} \mathrm{bw})$ as standard. Oral administration of methanolic extract of MEHP bark was conducted in a dose of 300- and $600-\mathrm{mg} / \mathrm{kg}$ bw for 1-, 4- and 12weeks.

\section{Assessment of analgesic Activity}

Acetic acid-induced writhing method. Antinociception 'study was performed according to the protocol described by Fontenele et $_{\text {al. }}{ }^{12}$

Nociception was induced by injection (i.p.) of $0.1 \mathrm{ml} / 10 \mathrm{~g}$ acetic acid solution $(10 \mathrm{ml} / \mathrm{kg})$. Test animals were grouped into four containing 6 rats each, where negative control and positive control groups were administered with distilled water and indomethacin $(10 \mathrm{mg} / \mathrm{kg}$, i.p. $)$ consecutively 30 minutes before acetic acid. The rats were pretreated with methanolic extract of $H$. pubescence stem bark at $300-$ and $600-\mathrm{mg} / \mathrm{kg}$ i.p. 30 minutes before acetic acid. Five minutes after the i.p. injection of acetic 
acid, the number of abdominal constriction (writhing responses) was counted up to next 10 minutes.

Formalin-induced writhing method. Test protocol described by Dubuisson and Dennis was followed with slight modification. Here5\% formalin in distilled water was prepared as pain inducing agent. Rats were grouped as negative control (distilled water), positive control (Indomethacin) and two extract groups (300 and $600 \mathrm{mg} / \mathrm{kgbw})$ containing 6 rats each. Each group was administered with their respected treatments before formalin injection in the dorsal surface of left hind paw. Writhing response was observed in two phases like 05 minutes (early phase) and 15-35 minutes (late phase). Results are expressed as percentage inhibition.

\section{Assessment of anti-inflammatory activity}

Carrageenan promoted rat paw inflammatory

method. Anti-inflammatory potential was measured by carrageenan induced edema method. Preweighted rats randomly divided into four groups carrying 6 rats each were delivered with their respected treatment with distilled water, indomethacin $(10 \mathrm{mg} / \mathrm{kg}), 300-$ $\mathrm{mg} / \mathrm{kg}$ bw and $600-\mathrm{mg} / \mathrm{kg}$ bw of MEHP. After 30 minutes of treatment, carrageenan $(1 \%)$ was injected into the rat paw to induce inflammation. Generation of inflammation (edema) was measured using plethysmometer at $0,30,60,120,180$ and 240 minutes of carrageenan injection. Data is presented as mean \pm SEM.

\section{Assessment of anti-diarrheal activity}

Castor oil induced anti-diarrheal test. Rats were selected by screening of their diarrheal response with $0.5 \mathrm{ml}$ of castor oil. Rats were taken in individual cages, the floors of which were lined with blotting paper and every hour, the floor lining was changed. Diarrhea was induced by oral administration of $0.5 \mathrm{ml}$ castor oil to each rat, 30 minutes before the above treatments. Every hour, total weight of fecal output, total weight of wet feces, total number of fecal output, and number of wet feces were recorded. Percent (\%) inhibition of diarrhea was calculated as follows:

(\%) inhibition of diarrhea $=$ (mean no. of wet defecation/mean wet defecation of control) $\times 100$

Statistical analysis, Graph Pad Prism (version 4.0) computer program (Graph pad Software San Diego, CA, USA) was used and the results are expressed as mean \pm SEM. A one-way analysis of variance (ANOVA), followed by Dunnett's post-hoc test or students paired or unpaired $t$-test were used in this study where needed.

\section{RESULTS AND DISCUSSION}

Preliminary phytochemical analysis. The result of qualitative chemical analysis of $H$. pubescence (MEHP) stem bark contained mainly alkaloids, glycosides, terpenoids, flavonoids, phenolics, tannins, anthraquinones etc. On the other hand carbohydrates, saponins, reducing sugars, proteins, vitamin $\mathrm{C}$ were not reported.

Blood glucose level. After administration of alloxan inexperimental rat, blood glucose level was significantly increased from $(18 \mathrm{mmol} / \mathrm{l})$ when compared with normal rats $(7.12 \pm .024 \mathrm{mmol} / \mathrm{l})$ (Figure 1A). After treatment for one week with standard drug glibenclamide, MEHP 300 and 600 $\mathrm{mg} / \mathrm{kg}$ bw respectively, glibenclamide, as a potential anti-diabetic drug, reduced blood glucose level to 7 . $56 \pm .16 \mathrm{mmol} / \mathrm{l}$ but MEHP failed to decrease blood glucose level.

On the other hand, in case of 4 weeks and 12 weeks treatment protocol, glibenclamide successfully reduced blood glucose level as $7.1 \pm 0.178 \mathrm{mmol} / \mathrm{l}$ and $6.1 \pm .145 \mathrm{mmol} / \mathrm{l}$, which is very significant but in case of 4 weeks treatment protocol, the methanolic bark extract of MEHP only showed minor reduction of blood glucose level and the values were $17.56 \pm$ 0.122 and $17.33 \pm 0.180 \mathrm{mmol} / \mathrm{l}$ for 300 and $600 \mathrm{mg}$ $/ \mathrm{kg}$ bw. For 12 weeks treatment protocol, the blood glucose level were reduced by only $2 \mathrm{mmol}$ and 3 $\mathrm{mmol} / \mathrm{kg}$ bw which are not significant (Figures 1B and $1 \mathrm{C}$ ). 


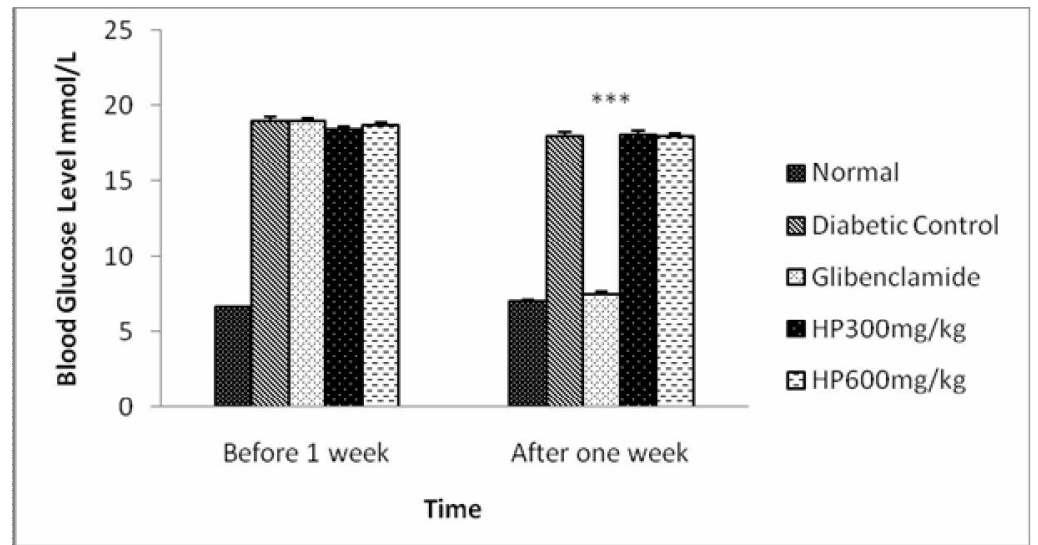

Figure 1A. Blood glucose level before and after one week $\left({ }^{* * *} \mathrm{p}<0.01\right.$ and $\left.\mathrm{n}=6\right)$ treatment.

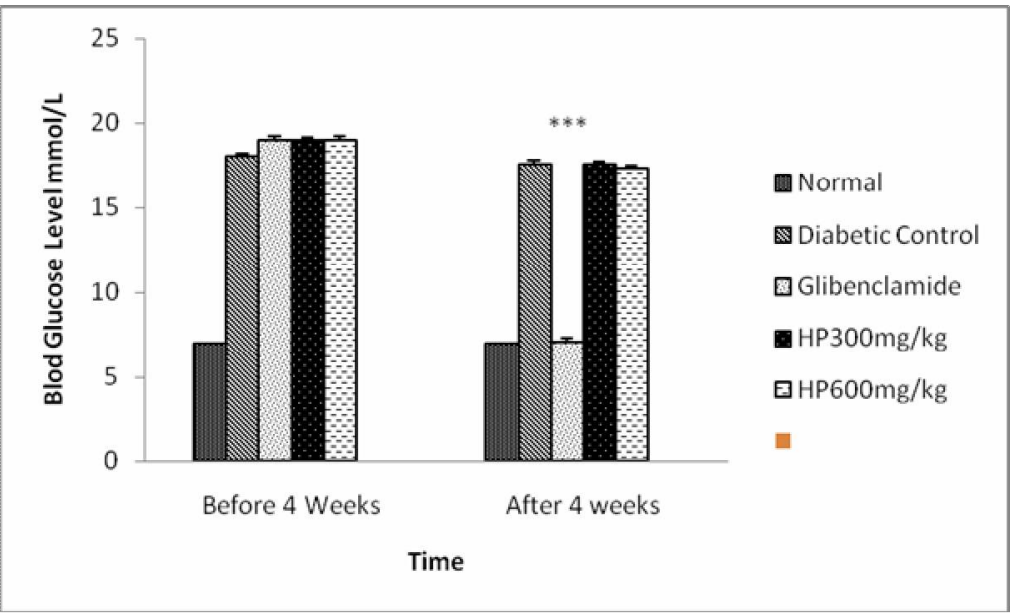

Figure 1B. Blood glucose level before and after four weeks $\left({ }^{* * *} \mathrm{p}<0.01\right.$ and $\left.n=6\right)$ treatment

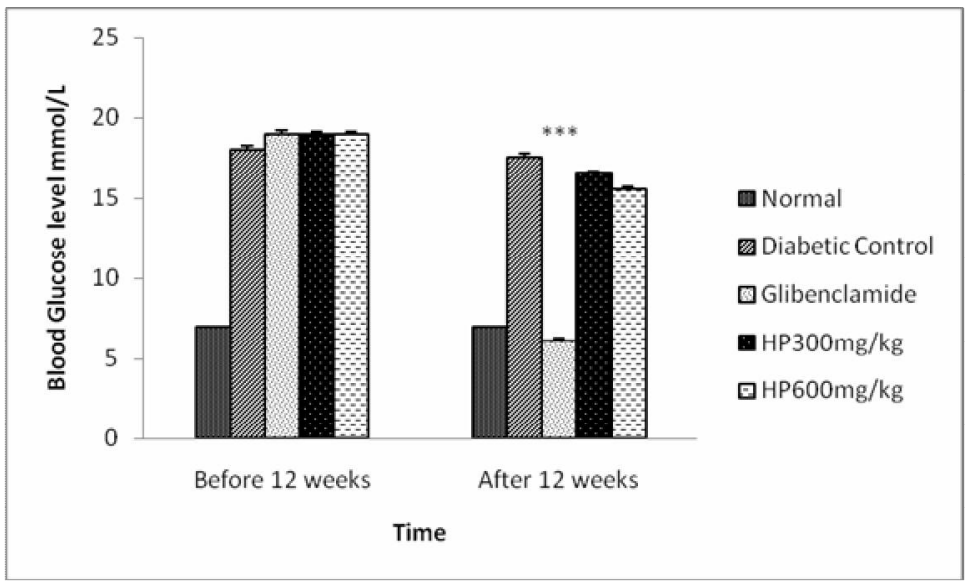

Figure 1C.Blood glucose level before and after twelve (12) weeks( ${ }^{* * *} \mathrm{P}<0.01$ and $\mathrm{n}=6$ ) treatment 
Acetic acid-induced writhing test. In acetic acid-induced method, MEHP showed inhibition of writhing response in dose dependent mannerwhen compared with distilled water (Figure 2). The MEHP at $\quad 600-\mathrm{mg} / \mathrm{kg}$ bwshowed strongly significant inhibition of writhing response $69.5 \%$ inhibition whereas standard indomethacin revealed $73.76 \%$ of inhibition.
Formalin-induced writhing test. As shown in figure 3, writhing responses were counted in two phases for formalin-induced analgesic protocol. In late phase, 300 and $600 \mathrm{mg} / \mathrm{kg}$ bw of MEHP demonstrated significant percentage inhibition 67.42 and $69.007 \%$, respectively. In addition, in early phase, only the higher dose of MEHP gave significant writhing inhibition (61.57\%).

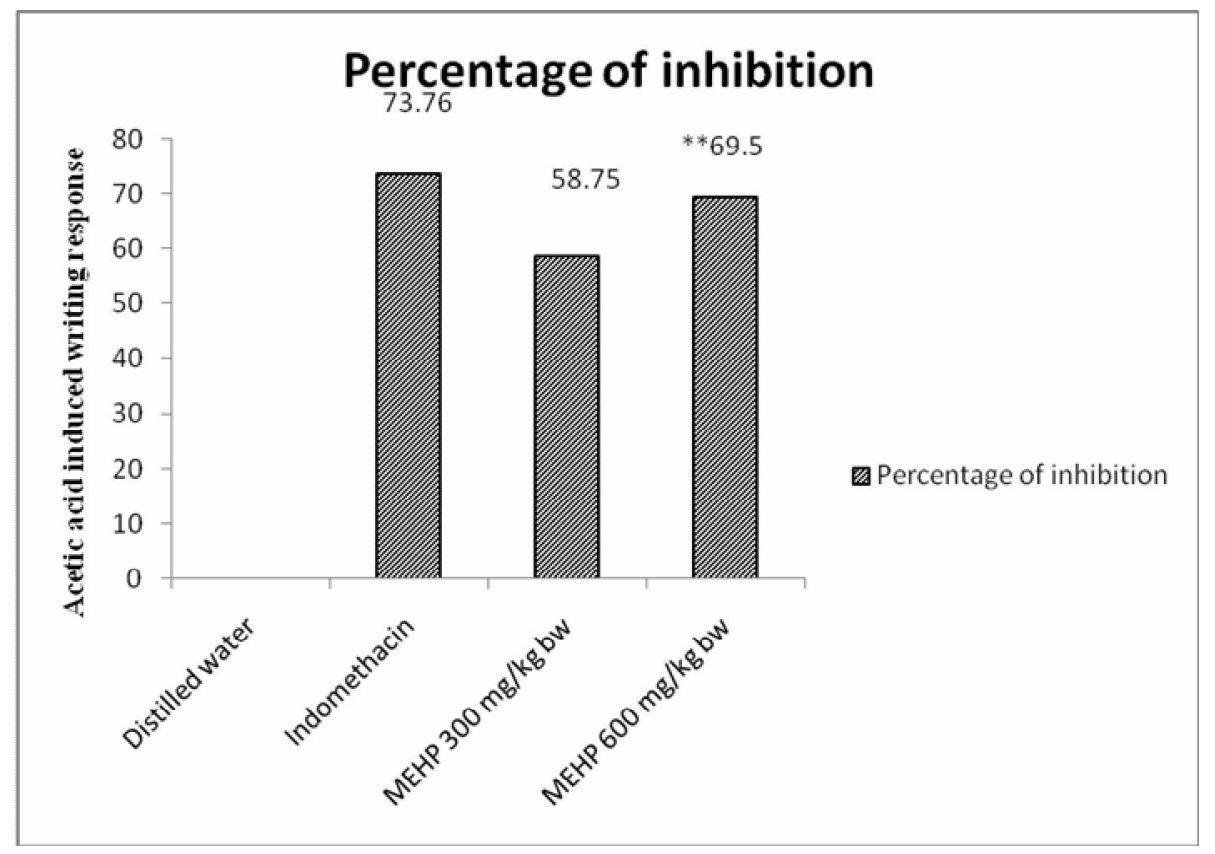

Figure 2. \% Inhibition of acetic-acid induced writhing response of $H$. pubescence in rat shown by MEHP $\left({ }^{* *} \mathrm{p}<0.01\right.$ and $\left.\mathrm{n}=6\right)$

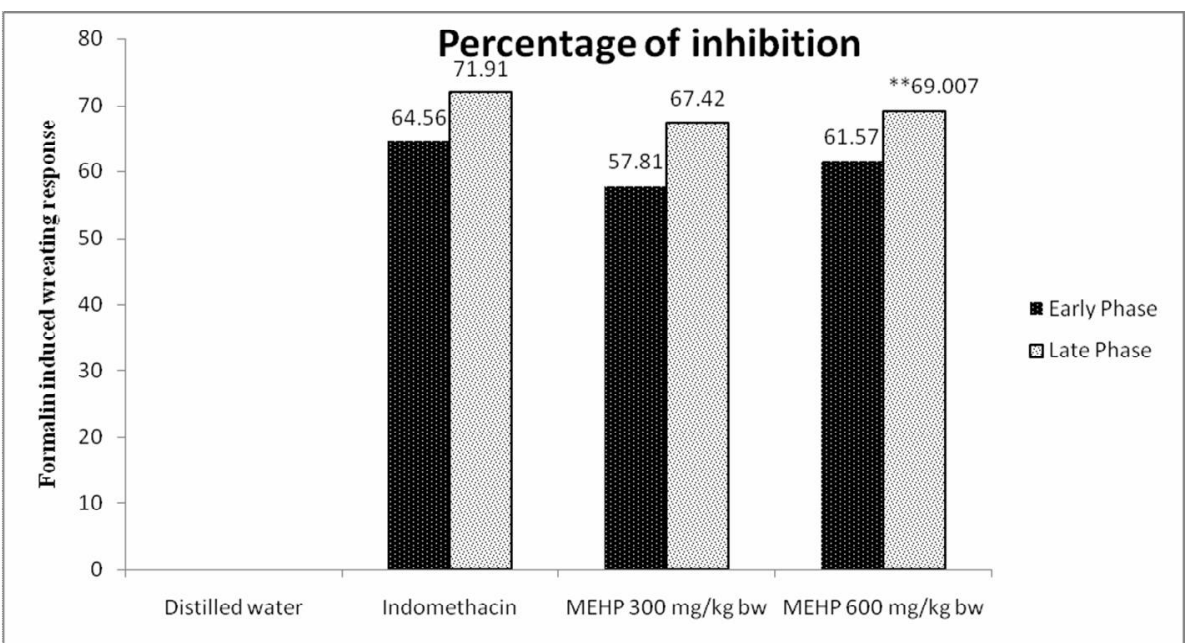

Figure 3. \% Inhibition of formalin-induced writhing response in rat by H. pubescence extract $\left({ }^{* *} \mathrm{p}<0.01\right.$ and $\left.\mathrm{n}=6\right)$ 
Anti-inflammatory study. In Table 1, antiinflammatory study of the MEHP extract reveals that higher dose showed slightly significant antiinflammatory potentials compared to the distilled water at 30 minutes and 60 minutes after carrageenan administration.
Anti-diarrheal potential assessment. The MEHP demonstrated significant anti-diarrheal potential in dose dependent manner. Figure 4 represented the percentage inhibition of fecation for MEHP where higher dose inhibited $71.43 \%$ and lower dose inhibited $64.92 \%$.

Table1.Data presenting paw thickness in carrageenan-induced anti-inflammatory study of MEHP.

\begin{tabular}{lcccc}
\hline \multicolumn{1}{c}{ Groups } & Distilled water & $\begin{array}{c}\text { Indomethacin } \\
10 \mathrm{mg} / \mathrm{kgbw}\end{array}$ & $\begin{array}{c}\text { MEHP } \\
300 \mathrm{mg} / \mathrm{kgbw}\end{array}$ & $\begin{array}{c}\text { MEHP } \\
600 \mathrm{mg} / \mathrm{kgbw}\end{array}$ \\
\hline \multicolumn{1}{c}{ Time interval } & & & \\
0 minute & $0.94 \pm 0.01$ & $0.90 \pm 0.06$ & $0.93 \pm 0.07$ & $0.92 \pm 0.04$ \\
30 minutes & $1.13 \pm 0.03$ & $0.98 \pm 0.04$ & $1.07 \pm 0.05$ & $1.02 \pm 0.06$ \\
60 minutes & $1.27 \pm 0.04$ & $1.06 \pm 0.05$ & $1.19 \pm 0.05$ & $1.09 \pm 0.06$ \\
120 minutes & $1.53 \pm 0.05$ & $1.21 \pm 0.07^{*}$ & $1.43 \pm 0.06$ & $1.36 \pm 0.05^{*}$ \\
180 minutes & $1.64 \pm 0.06$ & $1.37 \pm 0.06^{* *}$ & $1.59 \pm 0.07$ & $1.53 \pm 0.09^{* * *}$ \\
\hline
\end{tabular}

Values expressed as mean \pm S.E.M $(n=6)$. $(*)$ indicates statistically significant using one way analysis of variance, followed by Dunnett's multiple comparison test $\left(* p<0.05\right.$ and $\left.{ }^{* *} p<0.01\right)$

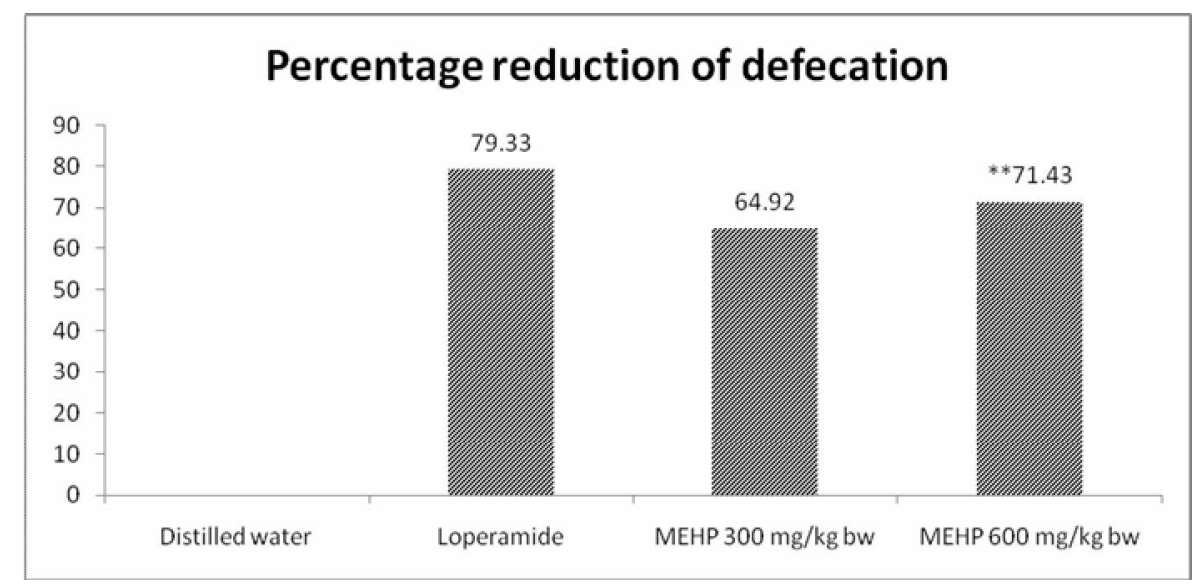

Figure 4. \% Defecation for castor oil induced anti-diarrheal method $\left({ }^{* *} \mathrm{p}<0.01\right.$ and $\left.\mathrm{n}=6\right)$

Our study showed that glibenclamide produced significant decrease in blood glucose level in alloxaninduced diabetic rats whereas MEHP failed to exert any significant effect in the reduction of blood glucose level. ${ }^{18}$ With the examination of analgesic activity, it can be summarized that standard indomethacin showed significant pain relieving potential that can be comparable with our tested plant. The MEHP samples demonstrated significant analgesic potential. It can also be assumed with their traditional use. Acetic acid specifically induce inflammatory pain. This test method is basically used to evaluate peripheral pain relieving potential. Early phase of formalin test indicates neurogenic pain and late phase represents pains which are controllable by NSAIDs. It revealed strongly significant analgesic potential at late phase. The MEHP showed strongly significant analgesic potential, whereas in inflammation it showed non-significant effect as antiinflammatory drugs. ${ }^{19,20}$ Previousstudies showed that presence of phytochemicals like terpenoids, flavonoids, phenolics and tannins are responsible for 
anti-nociception and anti-inflammatory potential. Anti-diarrheal study by castor oil demonstrated significant anti-diarrheal potential of MEHP in a dose dependent manner. It has been already established that phytochemicals present in the plant have anti-diarrheal potential and it supports previous studies. ${ }^{5-11}$

\section{CONCLUSION}

The methanolic extract $H$. pubescence of stem bark was found exibit significant analgesic and antidiarrheal activities by decreasing writhing responses and the intestinal fluid accumulation in rats. But antiinflammatory and anti-diabetic activities are not well reported. These can be possibly correlated with various phytoconstituents of the $H$. pubescence. However, further pharmacological studies are required to explore the exact mechanism of actions of the extract.

\section{REFERENCES}

1. Ghani, A. 1998. Medicinal Plants of Bangladesh: Chemical Constituents and Uses. Asiatic society of Bangladesh.

2. Chakraborty, A. and Brantner, A.H. 1999. Antibacterial steroid alkaloids from the stem bark of Holarrhena pubescens. J. Ethnopharmacol. 68, 339-344.

3. Patel, J.D., Patel, D.K., Shrivastava, A. and Kumar, V. 2008. Screening of plant extracts used in traditional antidiarrhoeal medicines against pathogenic Escherichia coli. Sci. World. 6, 63-67.

4. Verma, G., Dua, V.K., Agarwal, D.D., and Atul, P.K. 2011. Anti-malarial activity of Holarrhena antidysenterica and Viola canescens, plants traditionally used against malaria in the Garhwal region of north-west Himalaya. Malar. J. 10, 20.

5. Solanki, R., Madat, D., Chauhan, K., Khatiwala, B., Patel, H., Jignasu, M. and Adeshare, S.P. 2011. Evaluation of central nervous system activity of Holarrhena antidysenterica Linn. Apocynaceae bark. J. Phar. Res. 4, 1760-1761.

6. Jamadagni, P.S., Pawar, S.D., Jamadagni, S.B., Chougule, S., Gaidhani, S.N. and Murthy, S.N. 2017. Review of Holarrhena antidysenterica (L.) Wall. ex A. DC.: Pharmacognostic, pharmacological, and toxicological perspective. Pharmacogn. Rev. 11, 141.

7. Yang, Z.D., Duan, D.Z., Xue, W.W., Yao, X.J., and Li, S. 2012. Steroidal alkaloids from Holarrhena antidysenterica as acetylcholinesterase inhibitors and the investigation for structure-activity relationships. Life Sci. 90, 929-933.
8. Ali, K. M., Ghosh, A., Chatterjee, K., Mandal, S., Barik, B., Pathak, T. K. and Ghosh, D. 2011. Free radical scavenging activity of seed of Holarrhena antidysenterica: an in vitro study. J. Phar. Res. 4, 1631-1632.

9. Mana, S., Singhal, S., Sharma, N.K, and Singh, D. 2010. Hypoglycemic effect of Holarrhena antidysenterica seeds on streptozotocin induced diabetic rats. International J. Pharm. Tech. Res. 2, 1325-1329.

10. Ali, K.M., Chatterjee, K., De, D., Bera, T.K. and Ghosh, D. 2009. Efficacy of aqueous extract of seed of Holarrhena antidysenterica for the management of diabetes in experimental model rat: A correlative study with antihyperlipidemic activity. Int. J. Appl. Res. Nat Prod. 2, 1332.

11. Keshri, U.P. 2012. Antidiabetic efficacy of ethanolic extract of Holarrhena antidysenterica seeds in streptozotocininduced diabetic rats and it's influence on certain biochemical parameters. J. Drug. Deli. Ther. 2.

12. Khan, A., Khan, S. R. and Gilani, A. H. 2012. Studies on the in vitro and in vivo antiurolithic activity of Holarrhena antidysenterica. Urol. Res. 40, 671-681.

13. Khan, A., Bashir, S. and Gilani, A. H. 2012. An in vivo study on the diuretic activity of Holarrhena antidysenterica. Afr. J. Pharm. Pharmacol. 6, 454.

14. Ganapathy, P.S., Ramachandra, Y.L.. and Rai, S.P. 2010. Anti-inflammatory and analgesic activities of Holarrhena antidysenterica Wall. leaf extract in experimental animal models. Int. J. Bio. Pharm. Sci. 4, 101-103.

15. Ganapathy, P.S., Ramachandra, Y. L. and Rai, S.P. 2011. In vitro antioxidant activity of Holarrhena antidysenterica Wall. methanolic leaf extract. J. Basic. Clin. Pharm. 2, 175

16. Raman, M.S., Sultana, M. and Anwar, M.N. 2004. In vitro antimicrobial activity of halarrifine-24ol isolated from the stem bark of Holarrhena antidysenterica. Int. J. Agri. Bio. 6, 698-700.

17. Siddiqui, B. S., Usmani, S. B., Ali, S. T., Begum, S. and Rizwani, G. H. 2001. Further constituents from the bark of Holarrhena pubescens. Phytochem. 58, 1199-1204.

18. Begum, M.M., Sultana, Z., Ali, M.E., Jami, M.S.I., Khondkar, P., Khan, M.M. and Haque, M.M. 2014. Additive effect of lipid lowering drug (Simvastatin) in combination with antidiabetic drug (Glibenclamide) on alloxan induced diabetic rats with long term dyslipidemia. Indian J. Clin. Biochem. 29, 452-461

19. Huq, T.B., Rahman, M.S., Nure, M.A., Hossain, M.S., Sarwar, A., Islam, A. and Adhikary, B. C. 2017. Evaluation of pharmacological activities of methanol extract of Ixora bcuneifolia leaves. Clin. Phyto. 2, 22.

20. Hossain, M.S., Rahman, M.S., Imon, A.R., Zaman, S., Siddiky, A.B.A., Mondal, M. and Tabassum, A. 2017. Ethnopharmacological investigations of methanolic extract of Pouzolzia zeylanica (L.) Benn. Clin. Phyto. 2, 10. 